\title{
KMAUDITBR: UMA FERRAMENTA PARA DIAGNÓSTICO E AVALIAÇÃO DE SISTEMAS DE GESTÃO DO CONHECIMENTO
}

\section{KMAUDITBR: A TOOL FOR DIAGNOSIS AND EVALUATION OF KNOWLEDGE MANAGEMENT SYSTEMS}

\author{
João Pedro Albino ${ }^{1}$; Nicolau Reinhard ${ }^{2}$ \\ ${ }^{1}$ Universidade Estadual Paulista - UNESP - Bauru - Brasil \\ jpalbino@fc.unesp.br \\ ${ }^{2}$ Universidade de São Paulo - USP - São Paulo - Brasil \\ reinhard@usp.br
}

\begin{abstract}
Resumo
Muito se tem pesquisado e discorrido sobre a importância desempenhada pelo conhecimento nas organizações. Diante de um cenário de grande complexidade no mundo corporativo e na sociedade em geral, observamos que fenômenos econômicos e sociais, de alcance mundial, são responsáveis pela reestruturação do ambiente de negócios. A globalização da economia, e, sobretudo a dinâmica oferecida pelas tecnologias da informação e da comunicação (TIC's), apresentam uma realidade da qual as organizações modernas não podem lutar contra. É nesse contexto que a Gestão do Conhecimento (Knowledge Management) se transforma em um valioso recurso estratégico. A criação e a implantação de processos que gerem, armazenem, gerenciem e disseminem o conhecimento representam o mais novo desafio a ser enfrentado pelas empresas. Os sistemas de gestão do conhecimento (KMS - Knowledge Management Systems) buscam oferecer uma estrutura para impulsionar o compartilhamento do capital intelectual da organização de forma que os recursos investidos em tempo e tecnologia sejam efetivamente utilizados. Com o objetivo de propor uma infra-estrutura de diagnóstico e auditoria que permitisse avaliar a situação atual dos processos e atividades de uma organização foi desenvolvida a ferramenta KMAuditBr. $O$ objetivo desta ferramenta é o de proporcionar uma abordagem qualitativa para a avaliação das atividades e processos de Gestão do Conhecimento $(\mathrm{GC})$ internos à organização.
\end{abstract}

Palavras-chave: Gestão do Conhecimento (GC); Auditoria de GC; Ferramenta de Avaliação de Sistemas de GC.

\section{Introdução}

Muito se tem pesquisado e discorrido sobre a importância desempenhada pelo conhecimento nas organizações. Diante de um cenário de grande complexidade no mundo corporativo e na sociedade em geral, observamos que fenômenos econômicos e sociais, de alcance mundial, são responsáveis pela reestruturação do ambiente de negócios. A globalização da economia, e, 
sobretudo a dinâmica oferecida pelas tecnologias da informação e da comunicação (TIC's), apresentam uma realidade da qual as organizações modernas não podem lutar contra.

É nesse contexto que a Gestão do Conhecimento (Knowledge Management) se transforma em um valioso recurso estratégico. A criação e a implantação de processos que gerem, armazenem, gerenciem e disseminem o conhecimento representam o mais novo desafio a ser enfrentado pelas empresas. Os sistemas de gestão do conhecimento (KMS - Knowledge Management Systems) buscam oferecer uma estrutura para impulsionar o compartilhamento do capital intelectual da organização de forma que os recursos investidos em tempo e tecnologia sejam efetivamente utilizados.

Pesquisa realizada com executivos de grandes organizações mostrou que houve avanços na área, empresas investiram milhares de dólares para a implantação de processos de gestão do conhecimento em suas organizações, porém restam lacunas (gaps) a sobrepor (HSM, 200). Outros estudos recentes mostram que determinadas organizações ainda estão em processo incipiente no processo de desenvolvimento e implantação de projetos de gestão do conhecimento e muitas ainda gostariam de dar início a tais projetos.

Apesar de todo o acentuado interesse em gestão do conhecimento a principal questão a ser avaliada ainda é se a organização está compartilhando bem o seu conhecimento. Ou seja, como os centros de conhecimento identificados compartilham e gerenciam seu conhecimento? Torna-se importante o uso de ferramentas que possam direcionar os centros de conhecimento para as áreas que efetivamente exijam maior atenção além de identificar as práticas de gestão de conhecimento já em uso na organização, de forma que este conhecimento possa ser armazenado, mantido e distribuído de forma equânime.

Neste trabalho buscamos chamar atenção a um problema emergente nas quais os recentes estudos do fenômeno de compartilhamento do conhecimento não têm contemplado devidamente: a dificuldade entre o esforço para identificar os centros de conhecimento e desenvolver e implantar projetos de gestão de conhecimento nas corporações que sejam efetivamente utilizados.

O objetivo principal é o de propor uma infra-estrutura (framework) de auditoria que permita avaliar a situação atual dos processos e atividades da organização, visando prepará-la para diagnosticar sua real necessidade de gestão de conhecimento e sugerir referências para a elaboração de um modelo cujo objetivo seja o de aperfeiçoar e disponibilizar projetos de sistemas de gestão de conhecimento realmente úteis às organizações. 


\section{Definindo Conhecimento}

Conhecimento, segundo Nonaka e Takeuchi (1997) pode ser definido como uma "crença verdadeira justificada" e que apresenta semelhanças e diferenças entre conhecimento e informação. O conhecimento, segundo os autores, diz respeito a crenças e compromissos, sendo uma função de uma atitude, perspectiva ou intenção específica.

Além disso, o conhecimento, ao contrário da informação, está relacionado à ação. É sempre o conhecimento com algum fim. O conhecimento, assim como a informação, diz respeito ao significado. É específico ao contexto e relacional (ALBINO e REINHARD, 2006).

Davenport e Prusak (2003) definem a gestão do conhecimento como uma coleção de processos que governa a criação, disseminação e utilização do conhecimento para atingir plenamente os objetivos da organização.

Segundo estes autores, dados são um conjunto de fatos (atributos ou símbolos) distintos e objetivos, relativos a eventos. Em um contexto empresarial, os dados podem ser descritos como registros estruturados de transações. Informações são dados dotados de significado dentro de um contexto. No contexto empresarial, a informação pode ser descrita como um termo que permite a tomada de decisão e execução de uma ação, devido ao significado que tem para aquela empresa.

O conhecimento é derivado da informação, da mesma maneira que informação deriva de dados. De uma maneira simplificada, Davenport e Prusak (2003) consideram que um indivíduo gera conhecimento a partir da interação de um conjunto de informações obtidas externamente a ele, mas, também, com o conhecimento e informações já existentes em seu cérebro.

A construção do conhecimento é um esforço multifacetado, afirmam Albino e Reinhard (2005). De forma simplificada, requer uma combinação de ações sociais e tecnológicas. Na Figura 1 pode-se visualizar um modelo de gestão de conhecimento. Para uma empresa construir capacidade estratégica em conhecimento, a proposição é a de que pelo menos quatro componentes precisam ser empregados, ou seja: sistemas de conhecimento, redes de computadores, trabalhadores do conhecimento e organizações que aprendem. 
Figura 1 - Modelo de gestão do conhecimento.

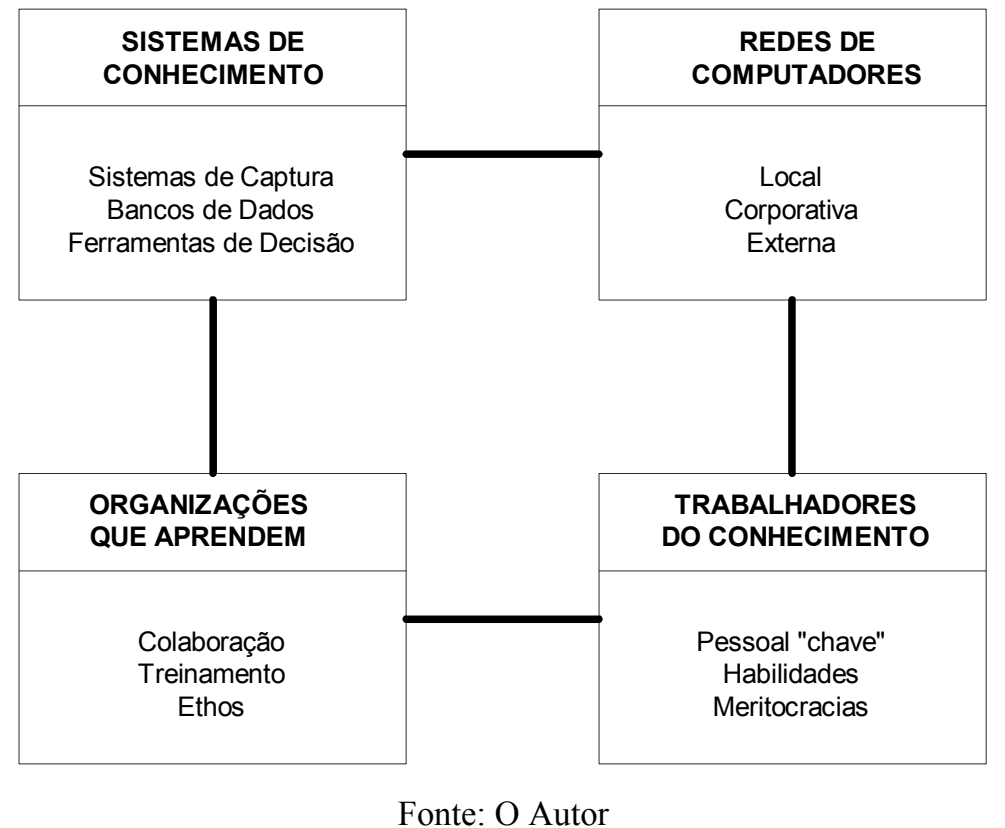

Nonaka e Takeuchi (1997) identificaram dois tipos básicos de conhecimento:

- Conhecimento tácito, ou implícito. Conhecimento pessoal, incorporado às ações e experiências dos indivíduos e específico ao contexto. Por envolver valores intangíveis, idéias, suposições, crenças e expectativas, além da forma peculiar de execução de atividades, é um tipo de conhecimento difícil de ser formulado e comunicado;

- Conhecimento explícito. Conhecimento articulado na linguagem formal, embutido em produtos, processos, serviços e ferramentas ou registrado em livros e documentos, sistematizado e facilmente transmitido, inclusive em afirmações gramaticais, expressões matemáticas, especificações, manuais, periódicos e assim por diante.

\subsection{Gestão do Conhecimento: Principais Fatores}

Segundo Awad e Ghaziri (2004) gestão do conhecimento é um modelo de negócio novo, emergente e interdisciplinar que tem o conhecimento como principal componente da estrutura organizacional. No seu tripé básico, a gestão do conhecimento é composta, segundo Awad e Ghaziri (2004), pela sobreposição de três fatores básicos: pessoas, tecnologia da informação e processos organizacionais (Figura 2). 
Figura 2 - Sobreposição dos fatores de Gestão do Conhecimento.

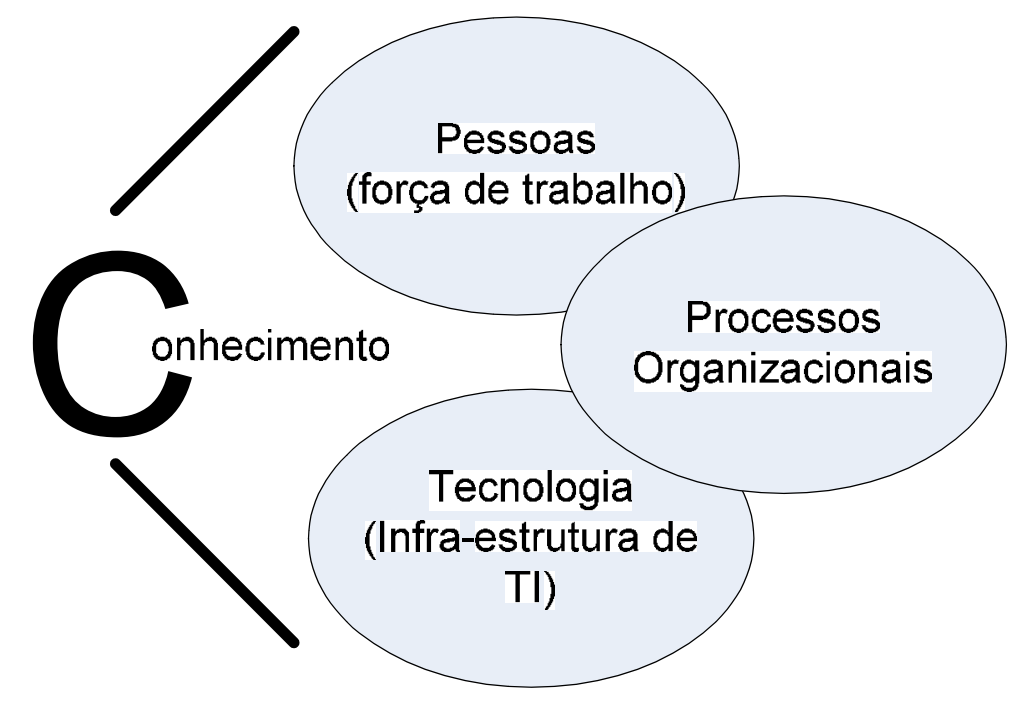

Fonte: Adaptado de Awad e Ghaziri (2004)

Um dos tripés das iniciativas de gestão de conhecimento, a Tecnologia da Informação (TI), trouxe grandes benefícios para as organizações. Novas tecnologias para comunicação com grande largura de banda, trabalho cooperativo e remoto, objetos e multimídia, ampliaram o ambiente informacional e hoje já existem inúmeras ferramentas que facilitam ou suportam os atuais projetos de gestão de conhecimento, segundo Albino e Reinhard (2006).

Tecnologias como portais corporativos de conhecimento $(P d C C)$, bases e mapas de conhecimento, software para discussão e chats eletrônicos, mapeamento de conhecimento tácito e explícito, data mining, gerenciamento de documentos, dentre outras tecnologias, já estão disponíveis e são oferecidas por vários fabricantes.

Uma taxonomia básica para ferramentas de Gestão de Conhecimento, pode ser visualizada na Figura 3. Segundo Awad e Ghaziri (2004), a estrutura conceitual da figura inclui as fases que tratam do ciclo de vida da gestão do conhecimento quais sejam: criação, coleta ou captura, organização, refinamento e difusão do conhecimento. 
Figura 3 - Taxonomia para Ferramentas de Gestão do Conhecimento.



Fonte: Adaptado de Awad e Ghaziri (2004)

A camada mais externa da Figura 3 representa o ambiente da organização - tecnologia, cultura, métricas e liderança.

Desta forma, tal estrutura tem grande influência em como a organização desenvolve e implementa seu ciclo de vida de gestão do conhecimento, o qual, segundo Awad e Ghaziri (2004), também pode ser definido como processo de gestão de conhecimento. Ainda segundo os autores, o último passo é a fase de manutenção, a qual garante que o conhecimento disseminado seja preciso, confiável e baseado nos padrões da empresa definidos a priori. Os principais tópicos desta estrutura são:

a) Adquirir: É o ato de prospectar, visualizar, avaliar, qualificar, fazer triagem, selecionar, filtrar, coletar, identificar;

a) Organizar/Armazenar: É o ato de explicitar, analisar, customizar, contextualizar e documentar;

b) Distribuir/Compartilhar: São os atos de disseminar, dividir, distribuir;

c) Aplicar: É o ato de produzir e usar;

d) Criar: É o ato de evoluir, inovar. 


\subsection{Ciclo de Vida dos Sistemas de Gestão do Conhecimento}

A construção da gestão do conhecimento pode ser vista, de acordo com Awad e Ghaziri (2004), como um ciclo de vida que se inicia com um plano mestre e uma justificativa e termina com um sistema estruturado para atingir os requisitos de gestão do conhecimento de toda a organização. Uma equipe de conhecimento representando as idéias da empresa e um desenvolvedor de conhecimento com experiência na captura, no projeto e na implementação do conhecimento garantem um sistema bem sucedido.

Segundo os autores, a fase mais crítica no ciclo de vida dos sistemas de gestão do conhecimento é a identificação das necessidades imediatas, intermediárias e de longo prazo para o sistema em prospecção.

Isto significa rever o núcleo de conhecimento dos funcionários; conduzir uma análise de custo e benefício para determinar a justificativa e os benefícios potenciais do sistema em planejamento; e determinar as ferramentas e os procedimentos para garantir a integridade, precisão e o sucesso operacional da implementação (AWAD e GHAZIRI (2004)).

Porém, antes da construção de um sistema de gestão do conhecimento torna-se necessária, segundo Tiwana (2000), a definição das principais fontes primárias e de qual a procedência do conhecimento para formar tal sistema. Desta forma, três passos básicos estão envolvidos no processo de conhecimento e aprendizado. Ainda de acordo com Tiwana (2000), observando estes três estágios pode-se abstrair qual o tipo de funcionalidade de tecnologia da informação irá apoiar este esforço. Estes três estágios compreendem:

a) Aquisição de conhecimento. É o processo de desenvolvimento e criação de idéias, habilidades e relacionamentos. As tecnologias de TI mais empregadas neste processo são ferramentas para captura de dados com capacidade de filtragem, bancos de dados inteligentes, escaners de teclado, captura de notes (anotações) e lousas eletrônicas;

b) Compartilhamento de conhecimento. Este estágio compreende a disseminar e tornar disponível o que já é conhecido. Este foco na colaboração e no suporte colaborativo são os principais fatores que diferenciam os sistemas de gestão do conhecimento dos sistemas de informação. Exemplos desta categoria de ferramenta são Lotus Notes da IBM e SharePoint da Microsoft;

c) Utilização do Conhecimento. A utilização do conhecimento ganha destaque quando o aprendizado está integrado à organização. Qualquer conhecimento que esteja disponível e sistematizado na organização pode ser generalizado e aplicado, pelo menos em parte, em uma nova situação. Qualquer infra-estrutura computacional disponível que apóie estas funções pode ser utilizada. 
Desta forma, utilizando este enfoque tecnológico estes três estágios e suas funcionalidades de tecnologia da informação estão representadas na Figura 4. Observa-se que esta figura é uma simplificação da Figura 3 vista anteriormente.

Figura 4. Estágios de utilização de conhecimento e suas funcionalidades de TI.

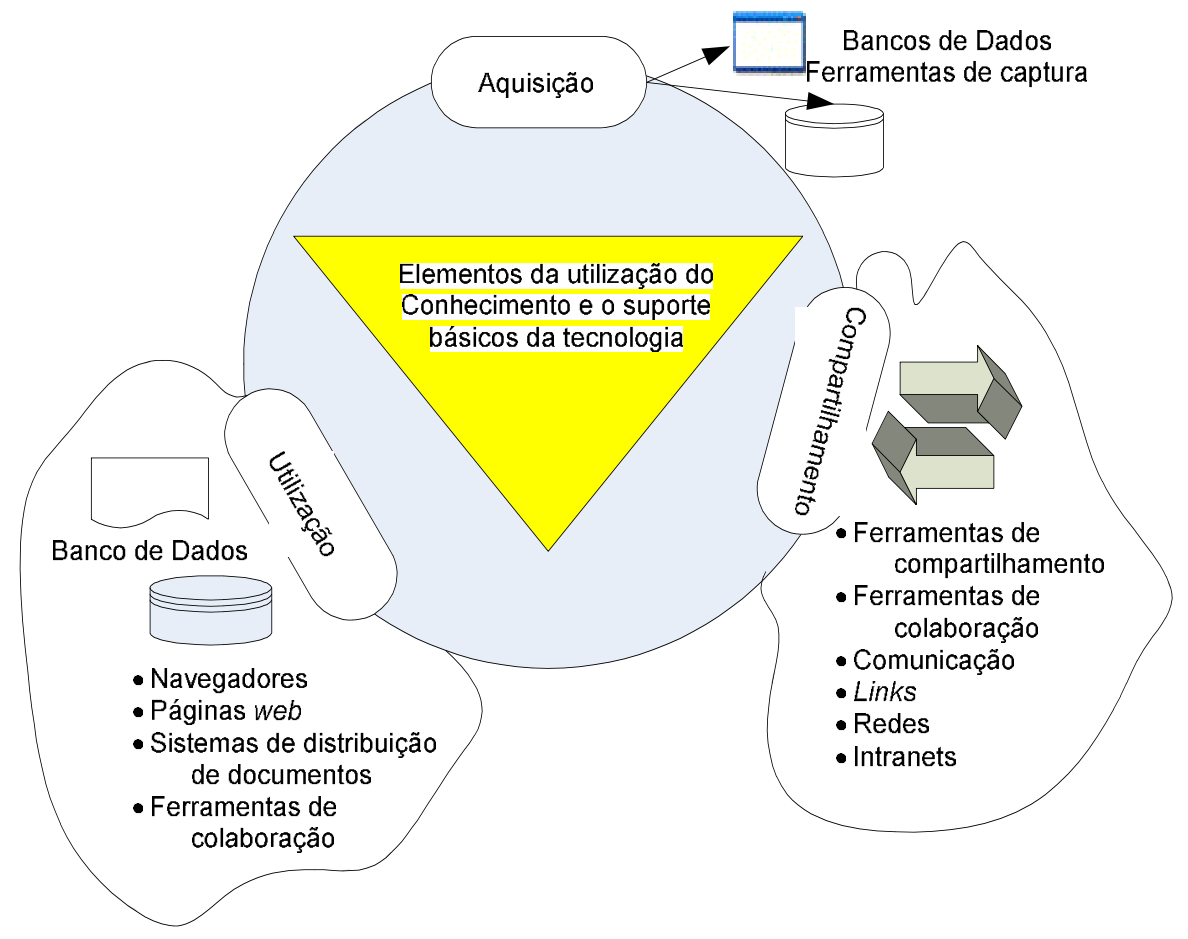

Fonte: Adaptado de Tiwana (2000)

De acordo com Tiwana (2000), estes três estágios não precisam estar em seqüência. Em algumas situações eles podem ocorrer em paralelo.

\subsection{Transferência de Conhecimento}

A verdadeira Gestão do Conhecimento, segundo Carmo (2003), não ocorrerá sem maiores mudanças nas abordagens de gerenciamento e na estrutura organizacional. De acordo com Garvin (1993), os programas de incentivo ao compartilhamento do conhecimento só serão bem sucedidos se os colaboradores sentirem que os benefícios do compartilhamento, através da experimentação excedem os custos, do contrário não participarão. Analisando diversos programas de educação e treinamento para o compartilhamento do conhecimento, o autor afirma que para assegurar o máximo de eficácia tais iniciativas devem estar diretamente conectadas com a implementação.

Se uma organização não possui uma cultura de compartilhamento será necessário trabalhar esta questão antes de trabalhar a estruturação da gestão do conhecimento, aconselham Gorelick, Milton e April (2004). 
A essência da maioria dos projetos de gestão do conhecimento é o compartilhamento de conhecimento, ou seja, é seguir o ciclo proposto por Davenport e Prusak (2003), que mencionam criação, disseminação e utilização de conhecimento. Se a empresa não possui um ambiente onde as pessoas compartilham informações e conhecimento, muitos dos projetos de gestão do conhecimento poderão ser prejudicados.

Mais importante que o gasto extraordinário em tecnologia e metodologias, de acordo com Gorelick, Milton e April (2004), seria a percepção generalizada dos valores estratégicos e competitivos a partir do conhecimento e conseguir com que os colaboradores, em todos os níveis da organização, passem a enxergar o compartilhamento do conhecimento como uma política da organização. Segundo Figueiredo (2003), o importante é disseminar entre os gerentes que antes do melhor software, os maiores aliados da gestão do conhecimento são a postura, a coerência, o respeito, as atitudes favoráveis e a própria estratégia do conhecimento.

\section{Auditando Gestão do Conhecimento}

A Gestão do Conhecimento (GC), segundo Keys (2006), oferece uma metodologia para criar e modificar os processos para promover a criação e o compartilhamento do conhecimento.

Porém, a compreensão do que é gestão do conhecimento organizacional ou corporativo ainda é relativamente lento, mesmo nas grandes organizações (HSM, 2004). Segundo Hylton (2002), mesmo empresas líderes de mercado que adotaram e implementaram GC, uma grande proporção falhou no processo ou não atingiu o sucesso esperado.

Ainda de acordo com Hylton (2002), existia no mercado a percepção que $80 \%$ das iniciativas de GC falhariam e que, grande parte dos projetos seriam abandonados após três anos do seu início. Isto levou, segundo a autora, à percepção de que as iniciativas de GC representariam uma aventura de alto rico.

Levando em consideração as questões apresentadas anteriormente, o promotor ou profissional de GC deve sempre procurar avaliar o estado atual da organização antes de iniciar um processo de gestão do conhecimento. Agindo desta forma, a estratégia dos projetos de GC seriam baseadas em evidências sólidas do estado atual das atividades e processos de GC e, a partir deste ponto, definir a melhor forma de implementar eficientemente gestão do conhecimento. Assim, haveria, segundo Hylton (2002), uma base sólida para determinar exatamente porque, como e onde os resultados associados aos benefícios seriam obtidos.

Segundo Bose (2004), os sistemas de Gestão de Conhecimento devem poder demonstrar o seu valor e se não houver sucessos que sejam mensuráveis o entusiasmo e o apoio para os projetos 
de GC provavelmente deixarão de existir. Portanto, se não existirem métricas e processos de mensuração sólidos, os administradores não poderão mostrar como os sistemas de GC funcionam, onde estão os gargalos e como este sistema pode ser melhorado.

Desta forma, de acordo com Hylton (2002), a auditoria deve ser a primeira fase ou etapa de uma iniciativa de GC e deve ser utilizada para fornecer uma investigação completa a respeito das políticas de informação e conhecimento, sua estrutura e seu fluxo. Uma avaliação completa, segundo a autora, deverá analisar o ambiente de conhecimento da organização, sua forma de compartilhamento e uso. Este processo permite também diagnosticar a cultura comportamental e social das pessoas dentro da organização, através da investigação da percepção das pessoas sobre a eficiência da gestão do conhecimento.

Segundo Rasmus (2001) uma ferramenta para avaliação e auditoria de GC deve incluir as seguintes áreas de investigação:

- Avaliação dos ativos intelectuais;

- Conhecimento como um ativo estratégico;

- O ambiente de colaboração;

- Cultura de aprendizagem interna;

- Cultura de compartilhamento de conhecimento;

- Importância do processo;

- Estrutura de comunicação;

- Motivação e iniciativa de recompensas.

Concluindo, Keys (2006) afirma que a característica mais importante a se considerar quando se define um instrumento de auditoria e avaliação é se o processo de mensuração mostra se o conhecimento está sendo compartilhado e utilizado. Para tanto, a avaliação deve estar ligada à maturidade da iniciativa de GC, a qual tem um ciclo de vida que progride através de uma série de fases.

\section{Uma Proposta de Ferramenta para Diagnóstico e Auditoria}

Com o objetivo de propor uma infra-estrutura de diagnóstico e auditoria que permitisse avaliar a situação atual dos processos e atividades de uma organização foi desenvolvida a ferramenta KMAuditBr.

A ferramenta KMAuditBr é um instrumento projetado para auxiliar as organizações a fazer uma avaliação inicial de alto nível de como está o processo de gerenciamento de conhecimento dentro da organização. 
O objetivo desta ferramenta é o de proporcionar, assim como o KMAT (Jager, 1999, e APQC, 2001) uma abordagem qualitativa para a avaliação das atividades e processos de Gestão do Conhecimento (GC) internos à organização. Ao completar todos os itens do instrumento a organização poderá ter uma visão panorâmica das áreas ou tópicos que requerem maior atenção, bem como identificar as práticas de gestão do conhecimento nas quais a empresa já apresenta excelência na execução.

\subsection{Estrutura da Ferramenta}

O modelo de estrutura do KMAuditbr é baseado no modelo de gestão de conhecimento apresentado na Figura 3, o qual propõe quatro facilitadores (liderança, cultura, tecnologia da informação e métricas) que podem ser utilizados para alimentar o desenvolvimento do conhecimento organizacional através do ciclo de vida de GC.

Este modelo coloca as principais atividades e os facilitadores de GC em um sistema único e dinâmico (JAGER, 1999).

Cada uma das partes do instrumento apresenta um conjunto de questões que permitem avaliar tanto como as práticas de GC estão com relação ao modelo quanto também coletar dados para avaliar seu desempenho e assim realizar um benchmarking com outras organizações.

A arquitetura básica do processo de auditoria e avaliação pode ser visto na Figura 5. 
Figura 5 - Modelo de arquitetura de GC

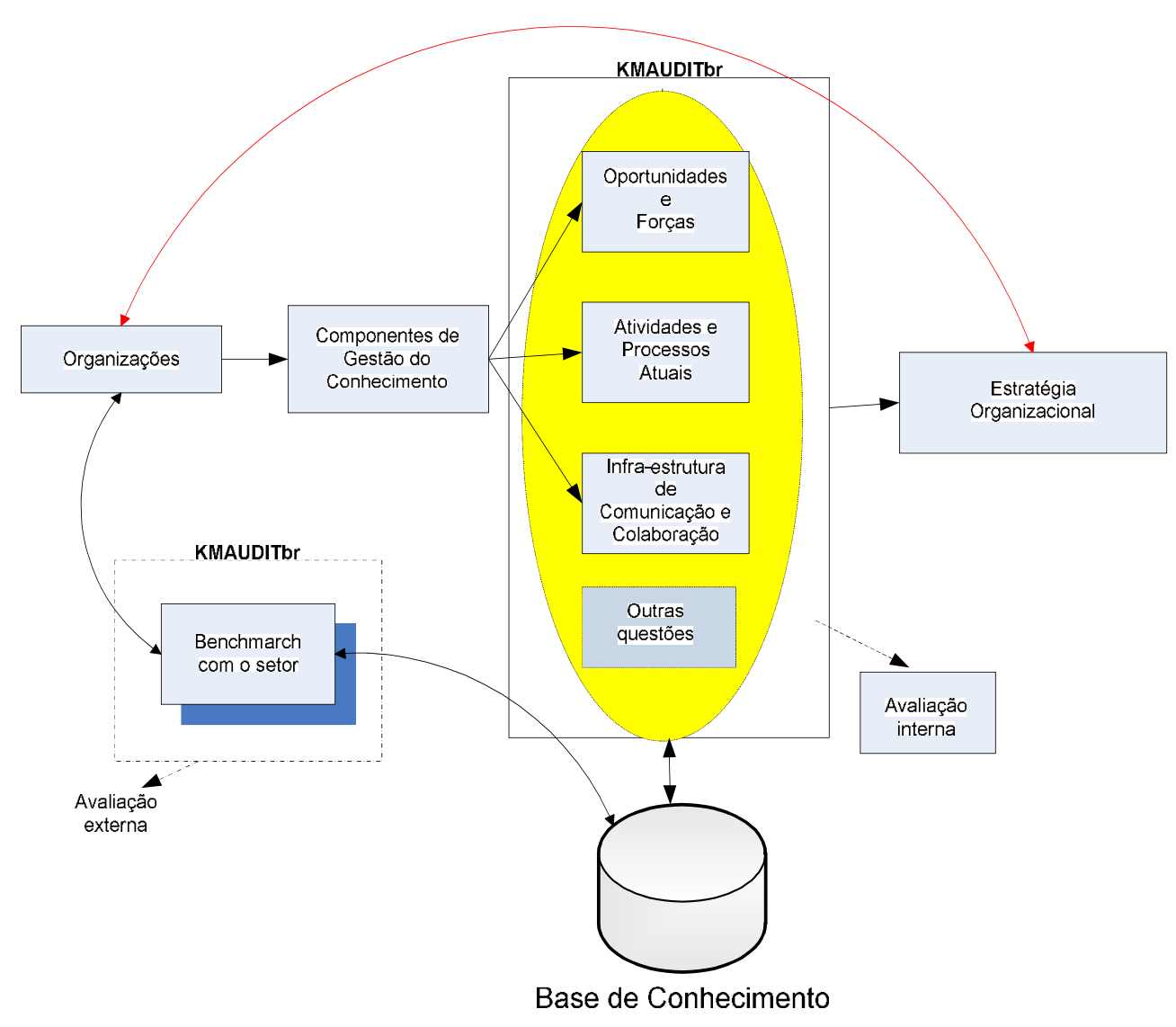

Fonte: do Autor

Baseado nos conceitos discutidos em Keys (2006), Hylton (2002), Rasmus (2001) e estendendo os conceitos apresentados no KMAT (Jager, 1999, e APQC, 2001), em resumo, esta estrutura apresenta os seguintes objetivos:

- Permitir realizar uma avaliação inicial de alto nível de gestão de conhecimento nas organizações;

- Avaliar como está o processo de gerenciamento de conhecimento dentro da organização;

- Proporcionar uma abordagem qualitativa das atividades e processos de GC internos através das medições proporcionadas;

- Permitir uma visão panorâmica das áreas ou tópicos que requerem maior atenção;

- Identificar as práticas de gestão do conhecimento nas quais a empresa já apresenta excelência na execução;

- Permitir benchmark externo com empresas do mesmo ramo.

Ao final do processo, a ferramenta permite posicionar qual o real estado da organização frente aos processos e atividades de GC em curso (Figura 6). Pensou-se, inicialmente, em quatro estados:

- Estacionado (ou parado). Existe um número ínfimo ou básico de processos ou nenhum; 
- Inicial. Onde as atividades e processos de GC são poucos;

- Priorizar e Selecionar. Um número grande de procedimentos está em curso, contado inclusive com apoio da TI para sua realização, porém sem coordenação ou um projeto coerente;

- Aprimorar e Continuar. Relação ótima entre processos de GC, projetos coordenados e uso de TI. Início do controle de qualidade para aprimoramento contínuo.

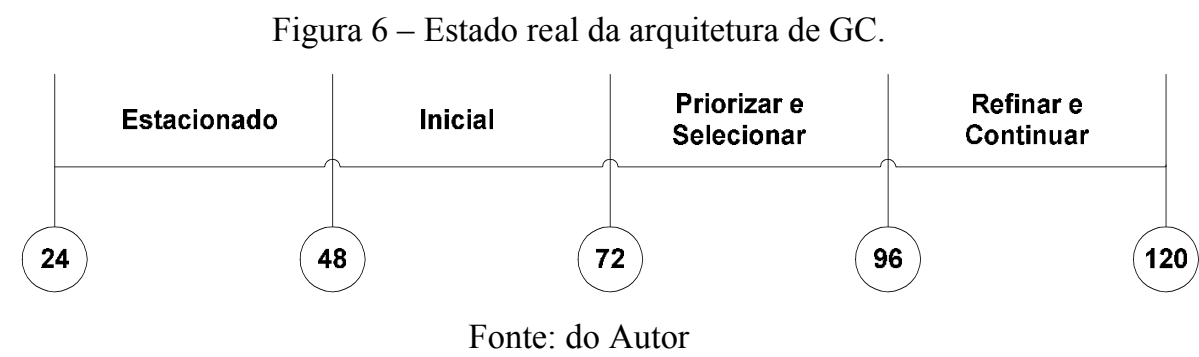

Por fim, após a realização de avaliações em organizações de vários setores e áreas de atuação, criar base de conhecimento das avaliações feitas e disponíbilizar, através do livre acesso à rede para os participantes no processo.

Desta forma, com as informações iniciais utilizadas para base de conhecimento, realizar um benchmarking colaborativo entre as organizações participantes de forma a criar uma estrutura onde um grupo de empresas compartilhe conhecimentos sobre uma determinada área de atuação, com o intuito de se aprimorar através do aprendizado mútuo.

\subsection{Detalhamento das Etapas de Diagnóstico e Auditoria}

$\mathrm{Na}$ parte inicial do processo de diagnóstico são coletadas informações à respeito da organização (Característica da Organização). São também coletadas informações à respeito do respondente do questionário (Característica do Indivíduo), questões relativas a sua percepção sobre Gestão do Conhecimento (Necessidade de Gestão do Conhecimento), bem como se existe na organização, segundo o conhecimento do respondente, investimentos em GC (setores ou áreas específicas, nível de investimento, etc.).

Estas informações iniciais, além de outras ao longo do questionário, são utilizadas para gerar uma base de dados que permite o desenvolvimento de um benchmarking colaborativo entre as organizações. Neste tipo de avaliação e comparação o que se busca é criar uma estrutura onde um grupo de empresas compartilhe conhecimento sobre uma determinada atividade, com o objetivo de se aprimorar através do aprendizado mútuo. 


\subsubsection{Avaliação das Forças e Oportunidades}

A segunda etapa do processo permite fazer uma análise profunda das atividades de GC na organização. Esta etapa se fundamenta em cinco seções, cada uma delas compreendendo um amplo espectro das atividades de GC.

Esta etapa permite auditar os seguintes itens:

- Processo. Inclui os passos de ação que uma organização utiliza para identificar a informação que necessita e a forma como coleta, adapta e transfere esta informação através da organização (verificação do fluxo da informação);

- Liderança. Compreendem questões de estratégia e como a organização define seu negócio e utiliza seus ativos de conhecimento para reforçar suas principais competências;

- Cultura. Reflete como a organização facilita o aprendizado e a inovação, incluindo nesta prática como encoraja os colaboradores a construir a base de conhecimento organizacional de forma a adicionar valor ao cliente;

- Tecnologia. Como a organização equipa seus membros para facilitar a comunicação entre eles, bem como da existência ou não de sistemas utilizados para coletar, armazenar e disseminar informação;

- Mensuração. Incluem não somente como a organização quantifica seu capital de conhecimento, mas também como os recursos estão alocados para fomentar o seu crescimento na organização.

Como foi apresentado, o objetivo desta segunda etapa é o de auxiliar as organizações a se auto-avaliar e verificar onde estão suas forças, fraquezas e oportunidades dentro do modelo de sistema de gestão de conhecimento da Figura 3 apresentado anteriormente.

\subsubsection{Avaliação das Atividades e Processos em Curso}

A terceira e última etapa busca realizar uma avaliação das atividades e processos em curso na organização que contemplem os estágios de utilização de conhecimento vistos anteriormente na Figura 4 (aquisição, compartilhamento, distribuição/utilização).

Os itens analisados nesta etapa permitem observar qual o tipo de funcionalidade de tecnologia da informação que está apoiando ou deveria apoiar o esforço de gestão de conhecimento. Além disso, permite avaliar também qual a estrutura de tecnologia da informação existente.

De acordo com Lindivall, Rus e Sinha (2003), a gestão do conhecimento deve ser apoiada por um conjunto de tecnologias para publicação eletrônica, indexação, classificação, 
armazenamento, contextualização e recuperação de informação, bem como para colaboração e aplicação de conhecimento.

Para dar conta das diferentes necessidades de aplicativos para gestão do conhecimento este trabalho tomou como base a arquitetura de sistema de gestão do conhecimento, delineada em Lawton (2001) e explicitada na Figura7.

Figura 7. Arquitetura de Sistema de Gestão do Conhecimento.

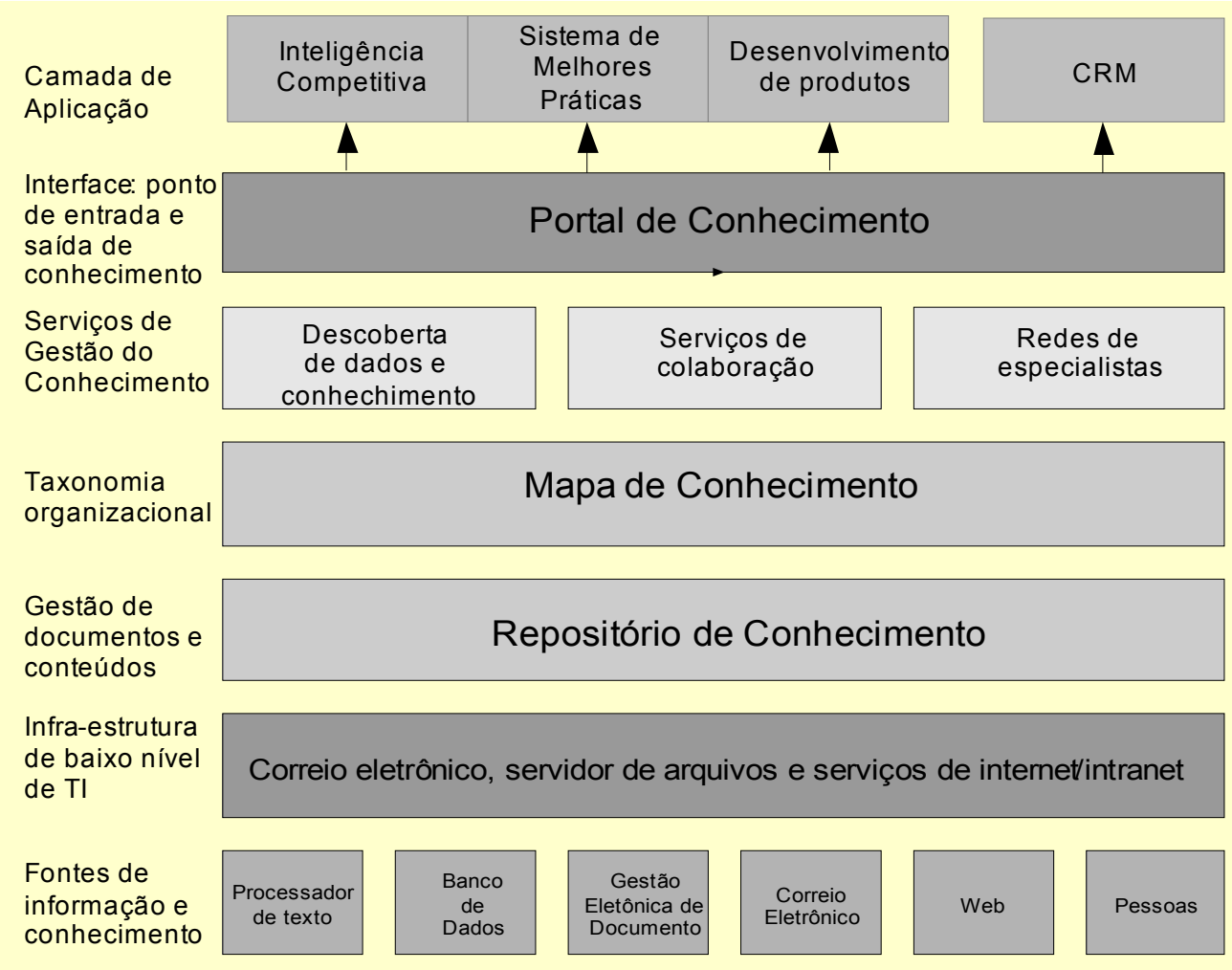

Fonte: Adaptado de Lawton (2001)

Desta forma, de acordo Lawton (2001),

Gestão de conhecimento não é simplesmente uma única tecnologia mas sim um conjunto composto de ferramentas para indexação, classificação e dispositivos para recuperação de informação acopladas com metodologias projetadas para obter os resultados desejados pelo usuário. (p.13).

As principais tecnologias disponíveis permitem, de acordo com Lawton (2001), que os trabalhadores do conhecimento trabalhem com gestão de conteúdo e de fluxo de trabalho (workflow), que categorizem o conhecimento e os direcionem as pessoas que possam se beneficiar deles, além de numerosas opções para melhorar a gestão do relacionamento com os clientes (CRM), busca e descoberta de conhecimento, agilizar o processo do negócio, bem como ferramentas para a colaboração e o trabalho em grupo, dentre outros propósitos.

Os componentes desta arquitetura de sistemas para gestão do conhecimento, segundo Lawton (2001), são: 
- Fontes de Conhecimento Explícito. O conhecimento explícito reside nos repositórios como documentos ou outros tipos de itens de conhecimento (por exemplo, como mensagens de correio eletrônico ou registros nos bancos de dados). Ferramentas padrão para editoração (tais como editores de texto) e sistemas de Gerenciamento de Banco de Dados (SGBD - DBMS) dão suporte a esta primeira camada;

- Infra-Estrutura de TI de Baixo Nível. Servidores de arquivo, programas de correio eletrônico (e-mails) além de serviços de intranet e internet dão suporte à camada de infra-estrutura de tecnologia da informação de baixo nível;

- Gestão de Documentos e Conteúdo. Sistemas de gestão de documentos e gestão de conteúdos são os aplicativos que fazem a manutenção da camada de repositório de conhecimentos;

- Taxonomia Organizacional. O conhecimento precisa ser organizado de acordo com o contexto de cada organização, baseada na taxonomia organizacional que cria um mapa de conhecimento, apoiado por ferramentas de classificação e de indexação;

- Serviços de Gestão do Conhecimento. Nesta camada as ferramentas que dão apoio a este nível são os sistemas de descoberta de conhecimento e as que oferecem serviços de colaboração;

- Interface e Camada de Aplicação. A distribuição de conhecimento na organização pode ser realizada através dos portais para diferentes usuários e aplicações tais como ensino a distância (e-learning), gestão de competências, gestão de propriedade intelectual e Gestão de Relacionamento com o Cliente (GRC - CRM).

O objetivo desta terceira etapa é o de auxiliar as organizações a se auto-avaliar e verificar quais são as atividades e processos de GC atualmente em uso e como a tecnologia da informação as está apoiando, dentro do modelo de sistema de gestão de conhecimento da Figura 3, utilizando como benchmarking a arquitetura de sistemas para gestão do conhecimento de Lawton (2001).

\section{Conclusões Preliminares}

De acordo com Rasmus (2001), as organizações bem sucedidas em Gestão do Conhecimento (GC) compartilham características comuns. Essas características vão desde a infra-estrutura de tecnologia a uma forte crença no valor do compartilhamento de conhecimento e da colaboração.

Pesquisas recentes (OECD, 2003 e HSM, 2004) têm demonstrado que as organizações que buscam desenvolver iniciativas de GC devem avaliar seu estado atual bem como um conjunto de vetores com as principais formas para determinar quais tipos de esforços de GC, derivados do seu 
processo de planejamento estratégico, provavelmente obterão sucesso. Os resultados da auditoria de GC podem assim ser utilizados para planejar as mudanças inevitáveis em tecnologia, processos e cultura organizacional que acompanham os investimentos realizados em GC.

As organizações que não avaliarem seu estágio de GC se arriscam, segundo Rasmus (2001), a correrem o sério risco de implementar tecnologias e conceitos em áreas onde o investimento será perdido ou não atingirá o objetivo proposto.

A ferramenta aqui apresentada, $K M A$ uditBr, foi idealizada com a finalidade de auxiliar a organização a fazer uma avaliação inicial de alto nível e avaliar como a mesma está gerenciando o conhecimento. O objetivo deste instrumento é o de orientar as organizações nas áreas que exigem maior atenção, assim como auxiliar a identificar as atividades e processos de GC que já estão em curso e desta forma, estimular o desenvolvimento do conhecimento organizacional. Questões como o suporte e o uso das funcionalidades da tecnologia da informação, também são avaliados pelo instrumento.

A avaliação oferecida pela ferramenta busca realizar um benchmarking interno, onde a empresa pode verificar se os seus objetivos e metas com relação à gestão do conhecimento estão sendo atingidos, e também um benchmarking externo, onde padrões do setor onde a organização atua poderão ser utilizados para adquirir um melhor aprendizado em GC e melhor se posicionar em relação às organizações similares. Outro objetivo do benchmarking é que a organização pode demonstrar o valor da função de gerenciamento de conhecimento em termos numéricos.

Não se pretende afirmar aqui que o $K M A u d i t B r$ seja uma ferramenta perfeita. Mas, assim como o KMAT (APQC, 2001 e Jager, 1999), de onde este instrumento é derivado, acreditamos que seja um passo inicial para gerar estudos mais aprofundados e mensuráveis sobre gestão do conhecimento. Busca-se com isso, sugerir que esta ferramenta seja utilizada como um instrumento de avaliação continuada a fim de permitir seu constante aprimoramento.

\footnotetext{
Abstract

Much has been researched and discussed about the importance played by the knowledge in organizations. Faced with a scenario of great complexity in the corporate world and in society in general, we observed that economic and social phenomena of global scope are responsible for restructuring the business environment. The globalization of the economy and especially the dynamic offered by information and communication technologies (ICT's) present a reality which the modern organizations cannot fight against. It is in this context that the Knowledge Management $(\mathrm{KM})$ is transformed into a valuable strategic resource. The creation and deployment of processes that generate, store, manage and deploy knowledge represent the latest challenge to be faced by companies. The knowledge management systems (KMS) seeks to offer a structure to foster the sharing of intellectual capital of the organization so that resources invested in time and technology are effectively used. With the objective to propose a framework to assess the current status of processes and activities of the organization that were developed a tool called KMAuditBr. The
} 
purpose of this framework is to provide a qualitative approach to evaluate knowledge management's activities inside the organization.

KeyWords: Knowledge Management, Knowledge Management Audit, Evaluation Tool of Knowledge Management Systems

\section{Referências}

ALBINO, J. P., REINHARD, N. Avaliação de Sistemas de Gestão do Conhecimento: Uma Metodologia Sugerida, In: XIII Simpósio de Engenharia de Produção - SIMPEP, Bauru, São Paulo. Anais do XIII SIMPEP 2006. Disponível em http://www.simpep.feb.unesp.br/upload/463.pdf.

Albino, J. P., ReInhard, N. A Questão da Assimetria Entre o Custo e o Benefício em Projetos de Gestão de Conhecimento. In: XI SEMINARIO DE GESTIÓN TECNOLÓGICA - ALTEC 2005, Salvador. Anais do XI Seminàrio de Gestión Tecnológica - ALTEC 2005.

APQC, Next-Generation Knowledge Management, Proceedings from APQC's 6th Knowledge Management Conference, American Center for Productivity and Quality, 2001.

AWAD, E. M., GHAZIRI, H. M. Knowledge Management, Prentice-Hall, USA, 2004.

BOSE, R., Knowledge Management Metrics, Industrial Management e Data Systems, V.104, N.6, 2004, pp. 457-468. cross ${ }^{\text {ref }}$

CARMO, R. M. Gestão do Conhecimento, Disponível em: www.informal.com.br/artigos, Acesso em: 01/11/2004.

DAVENPORT, T. H., PRUSAK, L. Vencendo com as Melhores Idéias: Como Fazer as Grandes Idéias Acontecerem na sua Empresa, Editora Campus, Rio de Janeiro, 2003.

FIGUEIREDO, S. P. Gestão do Conhecimento - Estratégias Competitivas para a Criação e Mobilização do Conhecimento na Empresa, Editora QualityMark,: 2005.

GARVIN, D. A. Building a Learning Organization, in Harvard Business Review on Knowledge Management, Harvard Business Review School Press, Boston, USA, 1993.

GORELICK, C., MILTON, N. APRIL, K. Performance Through Learning, Elsevier, USA, 2004.

HYLTON, A. A KM Initiative is Unlikely to Succeed Without a Knowledge Audit, Hylton Associates, April, 2002.

HSM, Gestão do Conhecimento na Prática, Revista HSM Management, N. 42, janeiro-fevereiro, 2004.

JAGER, M. The KMAT: Benchmarking Knowledge Management, Library Management, v.20, N.7, 1999, pp. 367372 .

KEYES, J. Knowledge Management, Business Intelligence, and Content Management: The IT Practitioner's Guide, Auerbach Publications, Taylor e Francis Group, LLC, New York, 2006.

LAWTON, G. - Knowledge Management: Ready for Prime Time?, IEEE Computer, Vol. 34, No. 2, pp. 12-14, 2001.

LIDVALL, M. RUS, I., SINHA, S. S. - Software Systems Support For Knowledge Management, Journal of Knowledge Management, Vol. 7, No.5, pp 137-150, 2003.

cross ${ }^{\text {ref }}$

NONAKA, I., TAKEUCHI, H. Criação de Conhecimento na Empresa: Como as Empresas Japonesas Geram a Dinâmica da Inovação, Rio de Janeiro: Campus, 1997.

OECD, Measuring Knowledge Management in the Business Sector: First Steps, Paris, 2003. 
RASMUS, D. W. Are You Ready For Knowledge Management? A Knowledge Management Assessment Tool, Planning Assumption, Giga Information Group, 2001.

TIWANA, A. The Knowledge Management Toolkit, Prentice-Hall, USA, 2000.

\section{Dados dos autores:}

Nome completo: João Pedro Albino

Filiação institucional: Faculdade de Ciências - UNESP/Campus de Bauru

Departamento: Computação

Função ou cargo ocupado: Professor Assistente Doutor

Endereço completo para correspondência (bairro, cidade, estado, país e CEP): Rua Salgado Filho, 6-69, Jardim Dona Lili, Bauru, São Paulo, Brasil, 17032-480

Telefones para contato: $0 * * 14$ 3103-6079; $0 * * 14$ 9792-0769

e-mail:jpalbino@,fc.unesp.br

Nome completo: Nicolau Reinhard

Filiação institucional: Faculdade de Economia, Administração e Contabilidade - FEA - USP/São Paulo

Departamento: Administração

Função ou cargo ocupado: Professor Titular

Endereço completo para correspondência (bairro, cidade, estado, país e CEP): Rua Professor Luciano Gualberto, 908 sala G122, SAO PAULO,SP, CEP: 05508-900

Telefones para contato: $0 * * 1130915838$

e-mail: reinhard@usp.br 